\title{
La era digital y la deshumanización a efectos de las TIC
}

Andrés García-Umaña - Pontificia Universidad Católica del Ecuador María Cristina Ulloa - Pontificia Universidad Católica del Ecuador

Évelyn Fernanda Córdoba - Pontificia Universidad Católica del Ecuador
0000-0003-2794-6069

D 0000-0002-7747-0030

0000-0001-5775-8558

Recepción: 05.06.2019 | Aceptado: 10.01.2020

Correspondencia a través de ORCID: Andrés García-Umaña

iD 0000-0003-2794-6069

Citar: García-Umaña, A, Ulloa, M y Córdoba, E (2020). La era digital y la deshumanización a efectos de las TIC. REIDOCREA, 9, 11-20.

Resumen: Las TIC son herramientas preferenciales que facilitan el acceso a la información y la comunicación, permitiendo multiplicidad de tareas. Sin embargo, el escenario podría estar desembocando en el aumento de la deshumanización en la sociedad. Objetivo: Analizar el nivel de acceso a las TIC de los profesores de educación pública de tres provincias del Ecuador -Manabí, Guayas y Santo Domingo de los Tsáchilas- y sus efectos colaterales. Método: Responder dos cuestiones: ¿La edad se relaciona con el nivel de habilidades digitales? y ¿Cuál es la perspectiva de los profesores frente a la deshumanización que han ido provocando las TIC en la sociedad? Resultados: Se respalda el enfoque de la investigación. Además, se determina que la edad es un factor influyente en la inserción de las tecnologías de la información dentro y fuera del aula de clases. Conclusiones: Por lo cual, existe la necesidad de estructurar políticas educativas enfocadas a la alfabetización mediática.

Palabra clave: Alfabetización digital

\section{The digital era and dehumanization for the purposes of ICT}

\begin{abstract}
In a world that travels hand in hand with technology, education along with educators needs to be constantly connected so as not to destabilize progress. ICTs are preferential tools that facilitate access to information and communication, allowing multiplicity of tasks. However, the scenario could be leading to an increase in the dehumanization of society. Objective: The present study aims to analyze $n=309$ public education teachers corresponding to three provinces of Ecuador -Manabí, Guayas and Santo Domingo de los Tsáchilas-, between 21 to $<62$ years old, with an average of 41.17 . Method: It focuses on analyzing in a quantitative way trying to answer two questions: Is age related to the level of digital skills? And what is the perspective of teachers against the dehumanization that has been causing ICT in society? Result: A literary review of the concepts that support the research approach is presented. It is also determined that age is an influential factor in the insertion of information technologies inside and outside the classroom. Conclusions: Therefore, there is a need to structure educational policies focused on media literacy.
\end{abstract}

Keyword: Digital literacy

\section{Introducción}

La investigación sobre las Tecnologías de la Información y Comunicación (TIC) podría tener su origen desde 1918, sin embargo, desde la década de los 50 s comienza a desarrollarse de forma más sistemática (Rangel, 2015).

A la par de la introducción de las TIC en la sociedad, desde diversas áreas, entre ellas la educación, se ha relacionado a estas con la deshumanización. Esto a partir de la idea de que las máquinas o artefactos han entorpecido las relaciones humanas, aislando a las personas y sometiéndolas al uso constante de dispositivos móviles o múltiples pantallas enlazados con diversos contenidos. Al estar en conexión con el mundo, a través de la red de Internet, diversos estudios concluyen que la sociedad en red o sociedad del conocimiento es la fuente de vida de la deshumanización. A la par, se defiende la idea de que la deshumanización es producto del ser humano a través de las tecnologías, no directamente de estas. 
En la educación por ejemplo, al conectar contenidos con el proceso de aprendizaje en red, omitimos la presencia física y el contacto interpersonal. Las conversaciones son más superficiales al no enriquecerse de elementos como el paralenguaje, la kinésica y la proxémica que generan experiencias profundas en la recepción de información. Los profesores ecuatorianos, comparten la idea de que las tecnologías forman parte del progreso de la sociedad, sin embargo su mal uso podría desencadenar consecuencias significativas. Ecuador es un país latinoamericano en proceso de desarrollo en muchos ámbitos, la presente investigación de aporte académico-científico nacional permite obtener una prospectiva de la relación existente entre el educando y la edad frente al uso de las tecnologías de la información, además socializar su perspectiva sobre los efectos deshumanizantes causados por las TIC.

\section{La era digital}

La convergencia tecnológica a mediados de los años 70, permitió el desarrollo de las computadoras. En el traslado de esta tecnología desde los sectores militares a la civilización en general, el efecto era evidente, el futuro se cristalizó, dando paso al surgimiento de los principios de la era digital (Bilbao-Osorio, Dutta y Lanvin, 2014). La definición de "era digital" hace referencia a un periodo de tiempo regulado por el uso constante de la tecnología. El término, moviliza capacidades y diferentes recursos que funcionan gracias a la combinación de conocimientos, significados e interpretaciones (Costa, Cuzzocrea y Nuzzaci, 2014). Por tanto, se podría afirmar que la evolución de la sociedad surge de manera acelerada, generando múltiples productos de comunicación e información.

Según De Casas-Moreno (2017) el desarrollo de las nuevas formas de comunicación informativas dio paso el aparecimiento de la sociedad de la información, siendo aquella en la que los conocimientos teóricos prevalecen y las fuentes de innovación emanan de la investigación y el desarrollo, así como de la nueva relación entre la ciencia y la tecnología.

El auge de las tecnologías en la sociedad, despierta el interés de múltiples investigadores, no solo por el potencial de crear dependencia, sino por la capacidad de fortalecer o debilitar los sujeciones entre las personas, de acuerdo al nivel de uso que se le pueda dar (Peñuela, Paternina, Moreno, Camacho, Acosta y De León, 2014). El contacto físico y la interacción cara a cara entre las personas ha cambiado, la comunicación ahora se realiza por medio de aparatos electrónicos o plataformas digitales (Umainman, Soheilian y Luu, 2018; Morozov, 2015). Según Aguaded (2005), la sociedad actual se define como un mundo en el que los medios de comunicación mediante lenguajes emergentes, forman el escenario actual.

El uso de las TIC en la era digital, ha permitido la ampliación de posibilidades de expresión de las personas en todos los ámbitos, evidenciando una oportunidad para generar ambientes de socialización, convivencia, aprendizaje y participación digital con responsabilidad (Castaño y Cabero, 2013). Las tecnologías digitales no crean comunidades, sino que contribuyen a la construcción de dinámicas sociales, existentes gracias a esfuerzos humanos (Roblizo y Cózar, 2015).

\section{La educación y la incidencia de las TIC}

En el ámbito educativo, se trata de explorar, comprender y adaptar las multifuncionalidades que ofrecen las TIC para mejorar el proceso de enseñanzaaprendizaje (Zambrano y Balladares, 2017; Unesco, 2015). Actualmente, se está incorporando un nuevo término denominado TAC (Tecnologías del Aprendizaje y el 
Conocimiento), que va más allá de aprender, apuesta por explorar herramientas tecnológicas al servicio del aprendizaje y de la adquisición del conocimiento (Rangel, 2015; Vázquez-Cano y Sevillano, 2015).

Ahora bien, actualmente se han incrementado múltiples propuestas y recursos educativos basados en TIC, entre ellos: cursos abiertos masivos en línea (Massive Open Online Courses-MOOC), el aprendizaje sin costuras (Seamless learning), el aprendizaje con el móvil (Mobile learning-Mlearning), los entornos de aprendizaje (Personal Learning Environments- PLE), entre otros; lo que permite a estudiantes y profesores alcanzar un acervo de herramientas e información en todos los campos de la educación para aprender, enseñar y compartir (García y Juanes, 2013).

Permanentemente se muestra más evidente que la relación entre el uso de Internet y los beneficios es compleja, pues depende de las habilidades y de otros muchos aspectos socio-demográficos, personales (Chadwick, 2013), culturales y estructurales (Bilbao-Osorio, Dutta y Lanvin, 2014). El aprendizaje en línea siempre deberá estar guiado por una serie de procedimientos técnicos y pedagógicos que permitan lograr un aprendizaje significativo. Roblizo y Cózar (2015), señalan que el responsable principal es el profesor, quien hace las veces de facilitador, intermediario e intercomunicador, entre las TIC y los educandos. Sin embargo, la lucha en contra de la analfabetización digital continúa, la brecha digital en muchos países sigue ejerciendo presión de apertura (Aesaert, Van Nijlen, Vanderlinde, Tondeur, Devlieger, y van Braak, 2015). Es el caso de los países emergentes, tales como Ecuador, Perú, Bolivia, Venezuela, entre otros (ITU, 2017; Banco Mundial, 2019; Johnson, 2016).

\section{Alfabetización mediática digital intergeneracional}

Entre los múltiples factores que han dificultado alcanzar niveles altos de alfabetización digital, se encuentra la brecha intergeneracional. En el camino de formación digital de los ciudadanos del siglo XXI, se ha visto la necesidad de adoptar metodologías de enseñanza para favorecer a la gestión de información. Sin embargo, más que realizar consumo de tecnología, es necesaria una formación mediática que potencie aspectos emocionales y actitudinales (Ferrés, Aguaded y García, 2012; Yayan, Düken, Dağ y Ulutaş, 2018).

Según Aguilar-Ramos y Urbano (2014), la presente sociedad requiere adultos con mente abierta a la actualización digital, reflexivos, con el deseo de dedicar tiempo a la auto capacitación. Pues sin duda, el Internet se ha convertido en fuente de aprendizaje (Tecnocimiento) a todos los niveles (Sandoval y Aguaded, 2012). No obstante, Internet mantiene una constante actividad que impulsa la desigualdad de oportunidades. El acceso material a la red se ha ido superando a nivel mundial, cada país invierte miles de dólares para reducir su propia brecha digital (ITU, 2017). Es posible que el material de acceso no sea suficiente para el crecimiento íntegro de la sociedad, cuando existen varios temas pendientes como la actitud de acceso, desarrollo de habilidades, beneficios y demás (Van Dijk, 2012).

Es decir que, la presente sociedad más allá de la resistencia de uso, requiere motivación de acceso e impulso de habilidades digitales. La edad no se puede convertir en un impedimento de aprendizaje, más bien en un estímulo de superación personal y adaptación de nuevas formas de aprender, enseñar y convivir (Apaolaza, He y Hartmann, 2014). El profesorado consiente de esta realidad, su desarrollo de habilidades digitales debe ser constante, con la finalidad de aportar al aprendizaje significativo de los estudiantes en materia de recursos digitales (Bartau, Aierbe y Oregui, 2017). 


\section{La deshumanización de la sociedad a efectos de las TIC}

Internet considerado para algunos autores como un hipermedio, al abarcar en este a varios medios y extender múltiples contenidos al alcance de todos a través de una conexión, ha acercado a las personas como una nueva forma de comunicación.

La humanidad se concibe entonces como una sociedad global que parte de elementos tecnológicos para la construcción de relaciones. Este fundamento es cuestionado por muchos autores, quienes debaten acerca del alcance de las TIC. Una de las posiciones tiene que ver con si estas permiten el avance a la humanidad, haciendo que más personas en todo el mundo vivan en mejores condiciones laborales, físicas y educativas; otra consiste en pensar, si por el contrario, la tecnología tiene un efecto deshumanizador, al pensar en sus consecuencias y constantes cambios asociados a la revolución digital (Argumosa-Villar, Boada-Grau, y Vigil-Colet, 2017).

Velarde y Ballesteros (2015) analizan a las TIC en cuatro grandes cuestiones: a) aspectos de la organización social; b) la toma de conciencia para el cambio social; c) mecanismos de control y d) relaciones interpersonales y conformación de las identidades. Respecto a los aspectos de la organización social, las autoras analizan que el intercambio de información en tiempo real afecta a la organización y al funcionamiento de la sociedad. Esta idea la refuerzan a través del pensamiento de Morozov (2015) quien manifiesta que Internet debilita el vínculo entre las organizaciones sociales y el territorio. Otros autores en cambio afirman que el intercambio cultural que se da a través de las redes, es necesario.

Wolton (2002), en sus diversos análisis, determina que las funciones que ofrece Internet constituyen "el principal triunfo de los sistemas automatizados". Afirma también que las TIC son mayormente aceptadas por los jóvenes debido a: "(...) la idea de apertura, el rechazo a la omnipresencia en los medios de comunicación de masas, el deseo de responder a la innegable angustia antropológica, la atracción por la modernidad y finalmente, la búsqueda de nuevas solidaridades con los países más pobres".

Wolton (2002) subraya que es necesario no confundir nueva tecnología y nueva cultura y advierte que debemos cuidarnos de mirar a la red como "soporte de una nueva solidaridad mundial". Sería entonces dicha afirmación la contraparte teórica para desestimar a "la toma de conciencia para el cambio social", factor que las autoras Velarde y Ballesteros (2015) consideran para la reflexión, como el elemento "humanista" de las tecnologías. Sin embargo, las TIC en su alcance, logran hacer visibles situaciones mundiales injustas a través de denuncias que circulan en la red de Internet. Estas exposiciones intentan promover respuestas solidarias a las desgracias colectivas.

En el intercambio de datos que se da en la red, también existe la generación de discursos manipuladores y falsos, que contribuyen a la desinformación. Esta consideración propondría una situación de análisis frente a la generación del caos y de la controversia, efectos que según Wolton (2002) "promueven el individualismo, devalúan la solidaridad y aíslan a los individuos".

En el análisis acerca de los mecanismos de control las autoras Velarde y Ballesteros (2015) analizan las críticas académicas a las TIC y concluyen, que según teóricos "estas facilitan mayor control de las instituciones sobre los comportamientos y las relaciones privadas; y al tiempo proporcionan autonomía a los ciudadanos" (2015, p.33). El control a los ciudadanos se ejerce hoy desde los espacios virtuales. Se ha recogido información importante para intereses de sectores vinculados a la política y las industrias sometiendo a la sociedad en un "estado de vigilancia" según Krug (2005). 


\section{Objetivos}

El presente estudio plantea el objetivo de analizar una población de profesores de educación pública para medir el nivel de habilidad en el uso de las TIC. Además, permitirá describir la relación de la edad y la frecuencia de uso de las tecnologías para la práctica docente. En suma, se pretende argumentar la perspectiva de la deshumanización a efectos de las TIC, respaldándolo en revisión literaria.

\section{Métodos}

\section{Participantes}

El público objetivo se eligió mediante un muestreo no probabilístico aleatorio simple (McMillan y Shumacher, 2005), de $n=309$ profesores de varias escuelas fiscales de tres provincias del Ecuador (Guayas, Manabí y Santo Domingo de los Tsáchilas), con 251 profesores mujeres $(81.3 \%)$ y 58 hombres $(18.7 \%)$.

\begin{tabular}{ll}
\hline Tabla 1. Descripción de la muestra & \\
\hline Provincia & $\mathrm{f}(\%)$ \\
\hline Guayas & $6(1.6)$ \\
Manabí & $218(70.6)$ \\
Santo Domingo & $84(27.2)$ \\
Total & $309(100)$ \\
\hline Edad & \\
\hline$<=21$ & $1(.3)$ \\
$22-31$ & $60(19.4)$ \\
$32-41$ & $101(32.7)$ \\
$42-51$ & $81(26.2)$ \\
$52-61$ & $55(17.8)$ \\
$62+$ & $4(1.3)$ \\
Total & $302(97.7)$ \\
Perdidos & $7(2.3)$ \\
\hline Total & $309(100)$ \\
\hline
\end{tabular}

\section{Instrumento}

La escala utilizada se ha validado de acuerdo al procedimiento de expertos. Basándose en las variables del enfoque de la investigación. Sosteniendo además una validación estadística con alfa de Cronbach $(\alpha=.86)$ aceptable para su implementación.

\section{Procedimiento}

El enfoque investigativo parte de lo cuantitativo a lo cualitativo, con la intención de no solo analizar los resultados numéricos, sino las diversas respuesta ideológicas en torno a una pregunta relacionada con la deshumanización de la sociedad a efectos de las TIC. La investigación mixta principalmente es un estudio exploratorio, que argumenta múltiples conceptos teóricos y señala autores que los respaldan.

\section{Análisis de datos}

Para la recolección de datos se realizó un evento formativo en el ámbito de las TIC, en donde asistieron los profesores y se proporcionó un cuestionario físico supervisado por los autores. Finalizado el proceso de recogida de información, los datos en bruto fueron 
exportados a una hoja de cálculo xls para su posterior importación y tratamiento estadístico en el software SPSS (Versión 22.0).

\section{Resultados}

En el presente apartado se describen los resultados alcanzados. Se ha procedido a agruparlos de acuerdo a las cuestiones de investigación. Partiendo de la relación existente entre la edad y el aumento o disminución de las habilidades digitales.

Pregunta 1: ¿La edad se relaciona con el nivel de habilidades digitales?

La edad del grupo de profesores intervenidos oscila entre 21 a $<62$ años, con una media de 41.17 y una desviación estándar moderada $(\sigma=9,918)$. Por su parte, el nivel de uso de las tecnologías se agrupó por escala de Likert representando los siguientes valores: 1=nulo, 2=poco uso, 3=uso medio, 4=medio alto, 5=alto. Los resultados descriptivos generales demostraron que los profesores de las tres provincias (Guayas, Manabí y Santo Domingo) sostienen una media de 3.01, con una desviación estándar aceptable $(\sigma=1.01)$.

\begin{tabular}{lcc}
\hline \multicolumn{2}{l}{ Tabla 2. Valores descriptivos sobre el nivel de uso de las tecnologías } \\
\hline $\mathrm{N}$ & Válidos & 309 \\
\hline Media & & 3.01 \\
Mediana & 3.00 \\
Desv. típ. & 1.010 \\
Mínimo & 0 \\
Máximo & 5 \\
Percentiles & & 2.00 \\
& 25 & 3.00 \\
& 50 & 4.00 \\
\hline
\end{tabular}

Por su parte, en la figura 1 se puede detallar el nivel de uso por provincia, según la edad. Describiendo que, la provincia del Guayas en una media $>40$ años sostienen un nivel de uso medio alto de las TIC. Por otro lado, en la provincia de Manabí, el mayor porcentaje de profesores $>47$ años de edad sostiene un nivel bajo y un reducido grupo de 33 años si se consideran diestros en el uso de las tecnologías. A su vez, en la provincia de Santo Domingo de los Tsáchilas, el grupo de profesores evidencia un manejo bajo de las TIC, de acuerdo a una edad media >50 años.

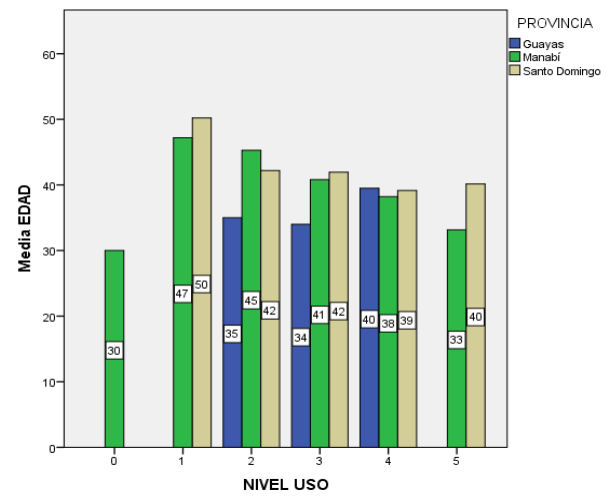

Figura 1. Nivel de uso según la edad y las provincias ecuatorianas 
A continuación, la tabla de contingencia describe según los grupos de edades el porcentaje con respecto al nivel de uso de las TIC. Resaltando que la gran mayoría de los profesores en las tres provincias se concentra en dos grandes grupos de edades de 32 a 41 años y de 42 a 51 años, señalando un preocupante $50 \%$ de este último grupo de poco nivel de uso de las tecnologías. No obstante, el grupo de edad de 22 a 31 años también registra un $41.7 \%$ en cuanto al desarrollo de habilidades digitales.

\begin{tabular}{|c|c|c|c|c|c|c|c|}
\hline \multirow{2}{*}{ Edad } & \multicolumn{6}{|c|}{ Nivel de uso f $\%$} & \multirow[t]{2}{*}{ Total } \\
\hline & 0 & 1 & 2 & 3 & 4 & 5 & \\
\hline \multirow[t]{2}{*}{$<=21$} & 0 & 0 & 0 & 0 & 1 & 0 & 1 \\
\hline & $0 \%$ & $0 \%$ & $0 \%$ & $0 \%$ & $1.4 \%$ & $0 \%$ & $0.3 \%$ \\
\hline \multirow[t]{2}{*}{$22-31$} & 1 & 0 & 7 & 25 & 19 & 8 & 60 \\
\hline & $100 \%$ & $0 \%$ & $11.3 \%$ & $19.4 \%$ & $27.1 \%$ & $40.0 \%$ & $19.9 \%$ \\
\hline \multirow[t]{2}{*}{$32-41$} & 0 & 3 & 18 & 45 & 27 & 8 & 101 \\
\hline & $0 \%$ & $15.0 \%$ & $29.0 \%$ & $34.9 \%$ & $38.6 \%$ & $40.0 \%$ & $33.4 \%$ \\
\hline \multirow[t]{2}{*}{$42-51$} & 0 & 10 & 21 & 36 & 13 & 1 & 81 \\
\hline & $0.0 \%$ & $50.0 \%$ & $33.9 \%$ & $27.9 \%$ & $18.6 \%$ & $5.0 \%$ & $26.8 \%$ \\
\hline \multirow[t]{3}{*}{$52-61$} & 0 & 7 & 15 & 21 & 9 & 3 & 55 \\
\hline & $0 \%$ & $12.7 \%$ & $27.3 \%$ & $38.2 \%$ & $16.4 \%$ & $5.5 \%$ & $100 \%$ \\
\hline & $0 \%$ & $35.0 \%$ & $24.2 \%$ & $16.3 \%$ & $12.9 \%$ & $15.0 \%$ & $18.2 \%$ \\
\hline \multirow[t]{2}{*}{$62+$} & 0 & 0 & 1 & 2 & 1 & 0 & 4 \\
\hline & $0 \%$ & $0 \%$ & $1.6 \%$ & $1.6 \%$ & $1.4 \%$ & $0 \%$ & $1.3 \%$ \\
\hline \multirow[t]{3}{*}{ Total } & 1 & 20 & 62 & 129 & 70 & 20 & 302 \\
\hline & $0.3 \%$ & $6.6 \%$ & $20.5 \%$ & $42.7 \%$ & $23.2 \%$ & $6.6 \%$ & $100 \%$ \\
\hline & $100 \%$ & $100 \%$ & $100 \%$ & $100 \%$ & $100 \%$ & $100 \%$ & $100 \%$ \\
\hline
\end{tabular}

Pregunta 2: ¿Cuál es la perspectiva de los profesores frente a la deshumanización que han ido provocando las TIC en la sociedad?

Por otra parte, se intenta dar respuesta analítica a la segunda cuestión de investigación planteada que responde al enfoque de pensamiento abierto sobre la incidencia de las Tecnologías de la Información y Comunicación en la deshumanización de la humanidad. Para ello, se concentran las respuestas obtenidas en tres grandes grupos con similitud de conceptos para facilitar el análisis interpretativo.

\begin{tabular}{lll}
\hline \multicolumn{3}{l}{ Tabla 4. Agrupación de respuestas abiertas - enfoque cualitativo } \\
\hline $\begin{array}{l}\text { Grupos } \\
\begin{array}{l}\text { G1: Pérdida de comunicación y } \\
\text { relación con la familia }\end{array}\end{array}$ & Provincia (f) & $\%$ \\
& Guayas (2) & .64 \\
& Manabí (60) & 19.43 \\
G2: Deshumanización & Santo Domingo (26) & 8.44 \\
& Guayas (3) & .97 \\
& Manabi (87) & 28.14 \\
G3: Dependencia & Santo Domingo (33) & 10.72 \\
& Guayas (2) & .64 \\
& Manabi (71) & 22.92 \\
& Santo Domingo (25) & 8 \\
& Total = 309 & 100 \\
\hline
\end{tabular}


Algunos comentarios socializados por los profesores intervenidos:

1. "No hay unión familiar ni diálogo"

2. "Prestan más atención a las TIC que a la vida real"

3. "Nos estamos convirtiendo en seres más superficiales"

4. "La tecnología aleja a las personas, es una lástima"

5. "La deshumanización se provoca por el mal uso de Internet"

6. "Que la sociedad da mayor tiempo al uso de las TIC descuidando los principios de humanidad se ha perdido las formas de respeto"

De acuerdo con la información obtenida, las personas encuestadas demuestran un alto índice de criterios comunes respecto a la relación del uso de la tecnología y la pérdida de comunicación y relaciones con la familia. Los datos obtenidos determinaron que el criterio de los profesores va en función de que las TIC afectan a las relaciones humanas fragmentando la comunicación y limitándola al contacto a través de medios móviles. De acuerdo a Peñuela, et al. (2014) "el gran auge que ha tenido esta tecnología ha suscitado inquietudes entre los investigadores, no solamente por su potencial de crear adicción sino por su capacidad de fortalecer o de debilitar los vínculos entre las personas, según el uso que se le dé al dispositivo". La tecnología ha desplazado los espacios presenciales por los virtuales, generando una reacción distante entre los miembros de una familia o de una comunidad.

A su vez, los profesores, a través de las encuestas realizadas, determinaron que las TIC influyen significativamente a la deshumanización, es decir que de acuerdo a sus criterios, las personas tienden a establecer tareas y acciones bajo la dependencia de los diferentes aparatos electrónicos, mermando su capacidad de análisis y de resolución frente a incertidumbres y conflictos presentados. Para los profesores, de acuerdo a las declaraciones recopiladas, es más fácil resolver consultas en Internet que razonar sobre una temática para emitir un criterio en base a lecturas y conocimientos adquiridos de forma tradicional.

Estos factores provoca la determinación de un tercer criterio, la dependencia hacia las TIC. Las personas, según los profesores consultados, tienden a empoderar a sus dispositivos dotándoles de valor significativo sobre sus vidas. Por ejemplo, el no soltar el móvil mientras los estudiantes están en clases o comparten una conversación, genera la sensación de apego hacia un aparato electrónico. De acuerdo al criterio de los profesores, mantenerse online todo el día, supone el desconectarse de la cotidianidad y de la concentración que deben mantener durante la recepción de información que reciben en los salones de clases.

En la actualidad, de acuerdo a Rush (2011) "los procesos de aprendizaje están totalmente influenciados por el uso intensivo de las tecnologías", este factor según los profesores encuestados, es relevante y positivo para la educación. Existe un criterio compartido entre la población de estudio que demuestra que sí existe influencia positiva de las TIC, siempre que se limite su uso y se condicione a estrategias en el marco del contexto de aprendizaje.

\section{Discusión}

En el marco de los resultados que arrojó la investigación realizada, se puede establecer que dentro de la muestra acogida la edad sí se relaciona con el nivel de habilidades digitales. Esto en función de que a mayor edad, menor uso de las TIC quizá debido a la falta de interés o desconocimiento. Este señalamiento es corroborado con las teorías antes expuestas, particularmente la determinación de que las habilidades y uso de la 
tecnología están determinadas por factores sociodemográficos (Inman, Soheilian y Luu, 2019).

La población que oscila entre los 31 a 51 años, usa medianamente y poco las TIC para la educación. Este factor evidencia la poca contribución de parte de los educadores de las zonas de estudio, a los procesos que la sociedad digital requiere. En el capítulo desarrollado en este texto, sobre "La educación y la incidencia de las TIC" se especifica la denominación de las tendencias que hoy se ejercen en esta área (la educación), las conocidas TAC (Tecnologías del Aprendizaje y el Conocimiento) (Rangel, 2015).

Los profesores de Ecuador manifiestan que le uso de las TIC de forma correcta benefician de forma relevante a la educación, sin embargo, cuando no existe un límite en su uso o una formación respecto a los contenidos, podrían generar fragmentación de las relaciones familiares, falta de comunicación, dependencia y la deshumanización. Se debe considerar entonces que "las relaciones sociales se están reconfigurando desde la aparición de las TIC. Desde luego se transforma el reparto del tiempo invertido en hacer cosas y en comunicar" (Surman y Reilly, 2005; García y Juanes, 2013).

Esto conlleva al criterio de que vivimos ante una generación que se transforma constantemente y que requiere de la formación constante de sus mentores. La orientación de los profesores hacia los estudiantes, ante estos cambios, implica el conocimiento de las herramientas tecnológicas, la no resistencia a los nuevos procesos educativos y la vinculación responsable de las TIC en los entornos de aprendizaje. Hoy, de acuerdo a Martín (2007) "los contactos virtuales sustituyen a los presenciales o los preparan (...) " por lo tanto, no se puede evadir las ventajas del uso de las TIC. Se debe sostener el criterio compartido de los profesores consultados en esta investigación de fomentar las buenas prácticas en el marco de su inclusión. Sin embargo, es responsabilidad de la familia y de las instituciones educativas buscar el monitoreo constante de dichas prácticas para proponer estrategias que promuevan la vigilia de la dependencia y de la deshumanización, así como el criterio extendido de que las herramientas tecnológicas generan la fragmentación de las relaciones y la comunicación.

El docente no debe sentir una limitación por su edad. La vocación de enseñanza debería llevarlo a aprender y compartir los conocimientos desde las innovaciones que a diario se dan en su área de formación. Queda también la necesidad de parte de los profesionales de la educación, de formarse en competencias de alfabetización digital con el objetivo de ir a la par de las consideraciones para fomentar una educación de mayor alcance y con miras a las generaciones que nacen o han nacido en el de la digitalización.

La implementación de las Tecnologías del Aprendizaje y el Conocimiento (TAC) debería ser una realidad en el Ecuador y en el mundo. Aquello consideraría un crecimiento sustancial en áreas pedagógicas. Sin embargo, no basta con implementarlo en las instituciones públicas o privadas, será necesario un aprendizaje regulado en padres de familia, profesores y público en general, mediante la implementación de políticas públicas de desarrollo social y cultural.

\section{Referencias}

Aesaert, K, Van Nijlen, D, Vanderlinde, R, Tondeur, J, Devlieger, I, \& Van Braak, J (2015). The contribution of pupil, classroom and school level characteristics to primary school pupils' ICT competences: A performance-based approach. Computer \& Education, 87(1), 55-69.
Aguaded, J. (2005). Estrategias de edu-comunicación en la sociedad audiovisual. Comunicar, 24(5), 1134-3478. 
Aguilar-Ramos, M. y Urbano, A. (2014). La necesidad de alfabetización digital e inter-generacional en la familia y la escuela. Didáctica, innovación y multimedia (DIM), 28(3), 1-16.

Apaolaza, V., He, J., y Hartmann, P. (2014). The effect of gratifications derived from use of the social networking site Qzone on Chinese adolescents' positive mood. Computers in Human Behavior, 41(3), 203-211.

Argumosa-Villar, L., Boada-Grau, J. y Vigil-Colet, A. (2017). Exploratory investigation of theoretical predictors of nomophobia using the Mobile Phone Involvement Questionnaire (MPIQ). Journal of Adolescence, 56(5), 127135.

Banco Mundial. (2019). Perspectivas económicas mundiales.

Bartau, I., Aierbe, A., \& Oregui, E. (2017). Parental mediation of the Internet use of Primary students: beliefs, strategies and difficulties. Comunicar, 54(1), 1-11.

Bilbao-Osorio, B. A., Dutta, S., y Lanvin, B. (2014). The Global Information Technology Report. Geneva: World Economic Forum

Castaño, C. y Cabero, J. (coords.) (2013). Enseñar y aprender en entornos de m-learning. Madrid: Síntesis.

Chadwick, A. (2013). The hybrid media system: Politics and power. Oxford University Press.

Costa, S., Cuzzocrea, F., \& Nuzzaci, A. (2014). Use of the Internet in Educative Informal Contexts. Implication for Formal Education. Comunicar, 43, 163-171.

De Casas-Moreno, P. (2017). La televisión sensacionalista en los canales públicos y privados en España e Italia. Estereotipos, discurso televisivo y los hábitos de la audiencia (tesis doctoral).

Ferrés I Prats, J., Aguaded Gómez, I., y García, A. (2012). La competencia mediática de la ciudadanía española. Competencias y retos. Revista de comunicación y tecnologías emergentes. ICONO14, 10(3), 23-42.

García, J. y Juanes, J.A. (2013). El cerebro y las TIC. Teoría de la Educación. Educación y Cultura en la Sociedad de la Información, 14(2), 42-84.

Inman, A. G., Soheilian, S., \& Luu, L. (2019). Telesupervision: Building bridges in a digital era. J Clin Psychol, 75(2), 292-301.

ITU (International Telecomunication Union). (2017). Measuring the information society report. Geneva, Switzerland: International Telecommunications Union.

Johnson, L. (2016). NMC Horizon Report: 2016 Higher Education Edition. Austin: The New Media Consortium.

Krug, G. (2005). Communication, technology and cultural cange. Sage. London-United Kingdom.

Martín, M (2007). Orígenes históricos de los usos actuales de la comunicación pública. Diálogos de la Comunicación, 69(1), 98108.
McMillan, J., y Shumacher, S. (2005). Investigación educativa. Madrid: Pearson

Morozov, E. (2015). La locura del solucionismo tecnológico. Madrid: Kartz Editores.

Peñuela, M., Paternina, J., Moreno, D. y Camacho, L., Acosta, L. y De León, L. (2014). El uso de los smartphone y las relaciones interpersonales de los jóvenes universitarios en la ciudad de Barranquilla (Colombia). Salud Uninorte, 30(3), 1-15.

Rangel, A. (2015). Competencias docentes digitales: propuestas de un perfil. Píxel-Bit. Revista de Medios y Educación, 46, 235248.

Roblizo, M. y Cózar, R. (2015). Usos y competencias en TIC en los futuros maestros de educación infantil y primara: hacia una alfabetización tecnológica real para docentes. Píxel-Bit. Revista de Medios y Educación, 47, 23-39.

Rush, S. (2011). Problematic use of smartphones in the workplace: an introductory study, Barts (Honours) thesis, Central Queensland University, Rockhampton.

Sandoval, Y. y Aguaded, I. (2012). Nuevas audiencias, nuevas responsabilidades. Revista de comunicación y tecnologías emergentes. ICONO14, 10(3), 08-22.

Surman, Mark y Reilly, K. (2005). Apropiarse de Internet para el cambio social. Hacia un uso estratégico de las nuevas tecnologías por las organizaciones transnacionales de la sociedad civil. Bilbao: Hegoa.

Umainman, A. G., Soheilian, S. S., \& Luu, L. P. (2018). Telesupervision: Building bridges in a digital era. Journal of Clinical Psychology, 75(2), 292-301.

Unesco (2015). Replantear la educación ¿Hacia un bien común mundial? Paris: Unesco.

Van Dijk, J. (2012). The evolution of the digital divide: Ther digital divide turns to inequality of skills and usage. Bus, Crompton, Hildebrandt y Metakides (eds.), Digital Enlightenment Yearbook, 2012, 57-75.

Vázquez-Cano, E. y Sevillano, M. L. (eds.) (2015). Dispositivos digitales móviles en educación. Madrid: Narcea.

Velarde, O. y Ballesteros, B. (2015). La implicación de las TIC en la humanización de la sociedad. Opción, 31(78), 31-41.

Wolton, D. (2002). Internet, ¿y después? Una teoría crítica de los nuevos medios de comunicación. Barcelona: Gedisa.

Yayan, E. H., Düken, M. E., Dağ, Y. S., \& Ulutaş, A. (2018). Examination of the relationship between nursing student's Internet and smartphone addictions. Journal of Human Sciences, 15(2), 1161-1171.

Zambrano, F. y Balladares, K. (2017). Sociedad del conocimiento y las TEPs. Innova Research Journal, 2(10), 169-177. 